\title{
Special issue on computational intelligence agents
}

\author{
Chang-Shing Lee $\cdot$ Vincenzo Loia
}

Published online: 5 August 2008

(C) Springer Science+Business Media, LLC 2008

The intersection between Computational Intelligence and Agent technology opens new significant scenarios in many fields where the representation and management of complex systems play a fundamental role. In the formulation of Agent-based systems, the role of uncertainty is crucial for an efficient and coherent resolution of complex problems. Agents overcome classical programs thanks to their inner capabilities to be autonomous and to adapt their behavior with the changing of the environment where agents live and interact. This means that inevitably they meet uncertainty during their work, or in many cases, for the high complexity of the problem, the information they handle is (or needs to be) approximate. In recent years there has been a growing awareness that Computational Intelligence handling of uncertainty in agents is equally important as other features of agent paradigm. The objective of the proposed special issue is to highlight an ongoing research on computational intelligence agents and their applications on various domains.

This special issue on computational intelligence agents receives thirteen papers, of which four papers have been accepted. This volume contains four papers that consider different aspects of perception based on the computational intelligent agents. The first paper HealthAgents: Distributed Multi-Agent Brain Tumour Diagnosis and Prognosis by Horacio Gonzalez-Velez, Mariola Mier, Margarida

\section{C.-S. Lee $(\bowtie)$}

Department of Computer Science and Information Engineering, National University of Tainan, Tainan, Taiwan

e-mail: leecs@mail.nutn.edu.tw

\section{Loia}

Department of Mathematics \& Computer Science, University of Salerno, Salerno, Italy

e-mail: loia@unisa.it
Julia-Sape, Theodoros N. Arvanitis, Juan M. Garcia-Gomez, Montserrat Robles, Paul H. Lewis, Srinandan Dasmahapatra, David Dupplaw, Andrew Peet, Carles Arus, Bernardo Celda, Sabine Van Huffel, and Magi Lluch i Ariet proposes an agent-based distributed decision support system for the diagnosis and prognosis of brain tumours developed by the HealthAgents project. The HealthAgents is a European Union funded research project and intends not only to apply forefront agent technology to the biomedical field, but also develop the HealthAgents network, a globally distributed information and knowledge repository for brain tumour diagnosis and prognosis.

The second paper Ontology-based Computational Intelligent Multi-Agent and Its Application to CMMI Assessment by Chang-Shing Lee and Mei-Hui Wang proposes an ontology-based computational intelligent multi-agent system for Capability Maturity Model Integration (CMMI). The computational intelligent multi-agent comprises a natural language processing agent, an ontological reasoning agent, and a summary agent. The multi-agent deals with the evaluation reports from the natural language processing agent, infers the term relation strength between the ontology and the evaluation report, and then summarizes the main sentences of the evaluation report. The results indicate that the proposed multi-agent can effectively summarize the evaluation reports for the CMMI assessment.

The third paper Inferring Threats in Urban Environments with Uncertain and Approximate Data: An Agent-based Approach by Lundy Lewis, John Buford, and Gabriel Jakobson discusses the problem of inferring threats in an urban environment, where the knowledge of the environment involves multiple types of intelligence and infrastructure data, and is by nature uncertain or approximate. The key innovations of the agent-based approach are an ontological commitment to events and situations, fuzzy event correlation, 
fuzzy situation assessment, adaptability and learning during threat assessment operations, and an enhancement of traditional belief-desire-intention (BDI) agents with situation awareness.

The last paper Adaptation of Proxy Certificates to NonRepudiation Protocol of Agent-based Mobile Payment Systems of Chung-Ming Ou and C. R. Ou presents an agentbased non-repudiation protocol based on mobile agents and proxy certificates. Non-repudiation of a mobile payment transaction ensures that when a buyer (B) sends some messages to a seller (S), neither B nor S can deny having participated in this transaction. An evidence of a transaction is generated by wireless public key infrastructure (WPKI) mechanism such that B and S cannot repudiate sending and receiving the purchase order respectively. The buyer gener- ates a mobile agent which carries encrypted purchase order to the seller and also issued a proxy certificate by the buyer. The advantage of this work is to reduce inconvenience for mobile clients such as connection time and provide a convenient way for mobile clients to reach non-repudiation for mobile payment transactions.

As guest editors of this special issue, we thank the authors for their contributions. We are most grateful to the referees for spending their valuable time in reviewing the manuscripts and providing kind cooperation and help. Finally, we greatly appreciated Professor Moonis Ali, the Editor-inChief, and the Applied Intelligence for providing us with the opportunity to edit and publish this special issue, as well as for their valuable instructions in the editorial process. 